\title{
Améliorer l'enseignement en grands groupes à la lumière de quelques principes de pédagogie active : penser à la méthode d'intégration guidée par le groupe (MIGG)
}

\section{Monsieur,}

C'est avec beaucoup d'intérêt que nous avons lu l'article de D. Vanpee, V. Godin et M. Lebrun, "Améliorer l'enseignement en grands groupes à la lumière de quelques principes de pédagogie active ", paru dans l'une des dernières livraisons du journal ${ }^{1}$.

Ce thème est tout à fait d'actualité car, comme l'expliquent les auteurs, en dépit des recommandations anciennes et régulièrement renouvelées de limiter les cours en amphithéatre, une part non négligeable des enseignements en sciences de la santé restent magistraux, en raison du nombre croissant d'étudiants à former, d'un effectif insuffisant en ressources enseignantes ou par manque de locaux adaptés.

Beaucoup parmi nous sont donc confrontés à la difficulté d'avoir à appliquer à des grands groupes d'étudiants les principes directeurs issus des sciences de l'apprentissage, et notamment de la psychologie cognitive, qui recommandent de mettre en cuvre une pédagogie active centrée sur l'apprentissage des étudiants.

Les auteurs proposent plusieurs pistes de réponse et suggèrent de recourir à plusieurs formats d'intervention pédagogique en grands groupes, qui permettent de solliciter explicitement l'activité des étudiants et de faciliter chez eux des apprentissages signifiants. Cependant, ils ne mentionnent pas la méthode d'intégration guidée par le groupe (MIGG), qui nous semble pourtant présenter beaucoup d'avantages à cet égard.

La MIGG est une technique pédagogique expositive, active et interactive, adaptée à l'apport de connaissances nouvelles. Codifiée depuis une quarantaine d'années par Daniel Chevrolet, psychopédagogue français qui a conduit plusieurs recherches et publications sur la dynamique des groupes de formation ${ }^{2}$, cette modalité pédagogique reste encore assez méconnue, peut-être en raison d'un acronyme "peu accrocheur". Nous en avions rapporté ici même une description opérationnelle sous forme de fiche pédagogique ${ }^{3}$.

La technique consiste pour le formateur à faire un exposé magistral court (inférieur à 20 minutes), avec support visuel. Le temps limité incite donc à sélectionner les informations pour atteindre les messages essentiels. Pendant toute la durée de l'exposé, il est demandé aux apprenants - consigne exceptionnelle - de ne pas prendre de notes. Ces derniers sont ensuite invités à restituer par écrit ce qu'ils ont retenu de l'exposé, entre les lignes du plan de cours préalablement fourni. Dans un second temps, les apprenants confrontent et complètent leurs notes avec leurs voisins de rang. A la fin de la séquence, le formateur réalise une synthèse avec le groupe pour s'assurer que le contenu a été "saisi" par le groupe. Ces étapes successives sont réalisables dans une grande salle de cours ou dans un amphithéâtre.

Notre expérience nous a permis de développer la MIGG auprès de publics très variés, étudiants sages-femmes, internes et médecins des équipes de service mobile d'urgence et réanimation (SMUR) et, plus récemment, auprès de formateurs en gestes et soins d'urgences (GSU). Initialement décrite pour des connaissances déclaratives, nous avons pu constater son efficacité pour préparer l'acquisition et l'organisation en mémoire de savoirs procéduraux. Par exemple, il est possible de développer une intervention pédagogique utilisant le format de la $M I G G$ sur le thème "réaliser l'examen clinique général et obstétrical d'une accouchée ", en amont d'une supervision clinique en maternité. Nous avons eu l'occasion de solliciter trente et un étudiants pour une évaluation qualitative à l'issue du cours. Trois d'entre eux connaissaient déjà la technique avant de rentrer à l'école de sages-fermmes. Tous se sont exprimés en faveur de la MIGG et se disent prêts à 


\section{Lettre à la rédaction}

renouveler l'expérience car elle rend le cours "plus clair, vivant, et ludique". Ils se sont perçus "plus concentrés, plus actifs, et moins " bêtes" ". La MIGG leur a apporté "une meilleure écoute, une meilleure mémorisation". Treize étudiants ont exprimé des difficultés : trous de mémoire compensés ensuite lors de la confrontation en sous-groupe (pour six d'entre eux), restitution discontinue avec des allers retours entre les lignes du plan (pour trois), peur de ne pas tout retenir (pour deux), difficulté de concentration (pour un), exposé trop long (pour un). Dixhuit étudiants ont déclaré n'avoir perçu aucune difficulté pendant le cours. Lors de la supervision en maternité dans les semaines qui ont suivi, nous avons pu constater une quête plus organisée et plus exhaustive des données cliniques auprès de la patiente.

Dans le cadre de la formation de formateurs en GSU, le Centre d'enseignement aux soins d'urgence (CESU) des Hôpitaux de Marseille sollicite la rétroaction des futurs formateurs GSU sur les enseignements dispensés. La MIGG que nous utilisons pour la formation à "l'accouchement inopiné " est évaluée par un score moyen de 4,45/5 par 195 formés qui ont bénéficié de cette technique dans les dix derniers mois.

Ces expériences montrent que la MIGG séduit par l'activité et l'interactivité qu'elle génère au sein du groupe, l'apport structuré et organisé des contenus, ainsi que son aspect ludique. Elle trouve sa place en formation initiale et continue, dès lors qu'il s'agit pour le formateur d'apporter des connaissances nouvelles. Elle se présente donc comme une alternative intéressante au cours magistral classique. Un recours plus large à cette technique serait susceptible d'améliorer les incontournables enseignements en grand groupe, tout en respectant les principes à présent bien connus de la pédagogie active.

Anne DEMEESTER

Directrice de l'Ecole de Sages-femmes de Marseille Faculté de Médecine Nord-Bd Pierre Dramard. 13916 Marseille cedex 20 Mailto:anne.demeester@ap-hm.fr

\section{Références}

1. Vanpee D, Godin V, Lebrun M. Améliorer l'enseignement en grands groupes à la lumière de quelques principes de pédagogie active. Pédagogie Médicale 2008;9:32-41.

2. Chevrolet D. Les méthodes pédagogiques actives. Angers : Institut de pédagogie et de recherche appliquée. [On line]. Disponible sur: http://www.ipraformation.com/methodes_ped.htm

3. Demeester A, Gagnayre R. Alternative au cours magistral : la MIGG (Méthode d'intégration guidée par le groupe). Pédagogie Médicale 2005;6:61-2. 